\title{
Cambios en las relaciones de trabajo: paradojas y consecuencias en el actual mundo del trabajo*
}

\section{Changes in work relationships: Paradoxes and consequences in the current world of work}

\author{
Verónica Andrade Jaramillo** \\ Pontificia Universidad Javeriana Cali, \\ Colombia.
}

Recibido: 2 de enero de 2013 Revisado: 30 de marzo de 2013 Aceptado: 10 de abril de 2013

\section{Resumen}

El objetivo del presente artículo es realizar una reflexión conceptual orientada a comprender los cambios acontecidos en el mundo del trabajo en las últimas décadas, por ser estos trascendentales para analizar la relación persona-trabajo. Dichos cambios se guían al menos a través de tres tendencias: la competición, la tecnologización y la globalización. Se mencionan las paradojas del actual mercado(s) de trabajo, y se explicitan seis cambios concretos producto de las tendencias y las paradojas; entre estos se mencionan la precarización del trabajo y el cambio en el funcionamiento de las organizaciones; los cambios en las formas y manifestaciones del trabajo; el cambio en la forma de representarse el ser humano al respecto del trabajo; los cambios en los criterios para la inclusión (y exclusión) y mantenimiento en el mercado de trabajo y la responsabilización del sujeto al respecto de su empleabilidad; los cambios en la configuración de la subjetividad y el lugar que el trabajo ocupa en ella incluyendo el establecimiento del contrato psicológico, la construcción de la carrera, la identidad, y la forma de

Correspondencia: Verónica Andrade Jaramillo (Candidata a Doctora en Psicología, Universidad del Valle) veroanja@hotmail.com. Dirección postal: Pontificia Universidad Javeriana Cali, calle 18 No. 118-250: Avenida Cañasgordas, Cali. Facultad de Humanidades y Ciencias Sociales, Edificio El Samán, Tercer Piso, Cali, Valle del Cauca, Colombia. 
representarse la agencia; y por último los cambios en la forma de gestionar el recurso humano al interior de las organizaciones. Se concluye que el estudio e intervención sobre el actual mundo del trabajo, requiere salir de visiones lineales y de causa-efecto, para contar con análisis reflexivos e intervenciones más pertinentes y atinadas, dada la complejidad que reviste hoy en día el mundo del trabajo.

Palabras clave: trabajo, competición, tecnologización, globalización, mercado.

\section{Abstract}

The aim of this paper is to make a conceptual analysis oriented to understand the changes occurring in the world of work in recent decades given that those changes are crucial to analyze the relationship between person and work. Such changes are driven by at least three trends: competition, technology and globalization. The paper makes a mention of the paradoxes of the current job market, and six specific changes are made explicit as a product of the above trends and paradoxes. Among the changes explained in the paper there are the rising of precarious jobs and the change in the organizations functioning; changes in the forms and manifestations of work; change in the way as human beings are represented with respect to the work; changes in the inclusion (and exclusion) criteria, stability in the labor market and the responsibility of every person about its employability; changes in the configuration of subjectivity and the place that the work occupies in such subjectivity, including the establishment of the psychological contract, the career construction, identity, and how to represent the agency; and finally the paper explains the changes in the way of managing human resources within organizations. We conclude that the study and intervention on the current world of work requires of leaving linear and cause-and-effect visions in order to consider thoughtful analysis and interventions more relevant and pertinent, given the complexity of nowadays world of work.

Keywords: Work, competition, technologization, globalization, market.

\section{Introducción}

Conocer las características del actual mundo del trabajo resulta fundamental para comprender el telón de fondo que sostiene -y explica- la relación persona-trabajo, en especial en este momento de la historia donde pareciera haber un entorno mundial convulsionado, heterogéneo y complejo.

Se parte de la tesis expuesta por Legge (1995) citada por Rentería (2009), al plantear que al respecto del mercado de trabajo -o del mundo del trabajo en general- aunque todo es diferente, nada ha cambiado. A través de la literatura especializada pueden rastrearse múltiples documentos que indican los cambios en el mercado de trabajo, donde algunos autores los presentan como cambios que transformaron radicalmente el panorama del trabajo comparado con décadas pasadas; sin embargo, es relevante indicar que el mercado de trabajo como se conoce basado en el capitalismo no ha sufrido propiamente cambios.

Al respecto de lo anterior, Antunes (1999) claramente lo indica, y aún hoy, después de varios años de su afirmación. Se concuerda con que es más pertinente hablar de la disminución profunda del trabajo estable asalariado y de la precarización del trabajo, que hacerlo sobre el fin del trabajo asalariado y del dominio del capital. Rentería 
(2009) respecto a lo propuesto por Legge (1995), explicita claramente lo que se mantiene y lo que cambia; en sus palabras:

Se mantienen las contradicciones históricas derivadas de la relación capital-trabajo, y entre tanto los repertorios, representaciones sociales, versiones, narrativas y sistemas de mediación simbólica presentes en las organizaciones de trabajo, y sobre el trabajo, han cambiado para legitimar lo correspondiente a cada época, de manera cada vez más sofisticada simbólicamente (p. 39).

Lo que cambia entonces tiene que ver concretamente con las relaciones de trabajo. El presente artículo tiene por objetivo comprender -más que listar- los cambios acontecidos en las relaciones de trabajo, por ser estos trascendentales para analizar la relación persona-trabajo. Se trata de caracterizar dichos cambios para entender el trasfondo al respecto de cómo las personas significan el trabajo, cómo lo viven, y la manera de moverse en este mundo o dimensión de la vida. Esto permitirá entender cómo las condiciones contextuales e históricas configuran las maneras en que se institucionaliza -o reinstitucionaliza- el trabajo, y afectan la relación persona-trabajo.

Antes de entrar al detalle con los cambios, resulta interesante mencionar lo que autores como Beck (2002), Malvezzi (1999), y Rentería (2009), et al. han dejado ver como las paradojas del actual mundo del trabajo que cada vez se radicalizan más, en la relación sucedida entre lo que ha cambiado y lo que no. A continuación se mencionan cinco paradojas que no agotan la descripción actual respecto a este tema en el mundo del trabajo.

En primer lugar puede mencionarse la paradoja referida a la vivencia de libertad vs. el abandono (Beck y Beck, 2002), donde cada vez las personas parecen sentirse más independientes, con mayores posibilidades de elegir a quién vender su fuerza de trabajo, y con mayor potencial para tomar decisiones respecto al mundo de trabajo libremente, pero al mismo tiempo están más desprotegidos que siempre, con menores posibilidades de tener seguridad social, con contratos a término fijo o indefino fácilmente diluibles, o con contratos por prestación de servicios donde el trabajador debe hacerse responsable de sí mismo totalmente en el sentido laboral (Peralta, 2012), por contar con la libertad de vender paralelamente su capacidad a otra organización o empleador, por solo mencionar un par de manifestaciones de esa desprotección, sin hablar de la cada vez más frecuente desprotección por parte del estado.

La segunda paradoja tiene que ver con la asunción de la existencia de la realidad vs. la creación de la misma a través del discurso. Esta consiste en que cada vez es más difuso tener claridad sobre qué es verdad y qué es una invención a través de las imágenes que se producen de las cosas que realmente sucedieron. Esta paradoja puede ser entendida con mayor profundidad a través de textos como el de Gergen (1991), quien al ilustrar las características de lo que denomina como postmodernismo, deja ver claramente la creación de nuevas realidades no existentes tiempo atrás, todas asentadas en la capacidad simbólica del ser humano, realidades que pese a no tener acento necesariamente en lo tangible, tiene resultados con efectos concretos en las personas y en la sociedad en general, como que los trabajadores acepten bajos salarios y condiciones desfavorables de trabajo, bajo el discurso de la crisis permanente (Peralta, 2012). Otro ejemplo de esta paradoja puede verse en el funcionamiento del mercado bursatil desde las crisis de los últimos años, en particular desde el 2008, donde algo estrictamente simbólico como este tipo de mercado, influye realmente sobre los niveles de pobreza o riqueza de las personas y los países, a través del mecanismo de la especulación.

Una tercera paradoja que puede mencionarse se relaciona con la necesidad de interdepender vs. el individualismo; por una parte se le señala al trabajador que cada vez más su inserción y mantenimiento en el mercado de trabajo depende de sí mismo, de sus capacidades y competencias, pero al mismo tiempo requiere de los otros y de las organizaciones para mantenerse enganchado en el mundo del trabajo. Asimismo, sin prometerle o asegurarle el futuro laboral, se le exige en el trabajo que se comprometa con la organización 
a la que le sirve, que se "ponga la camiseta", y a veces que trabaje de manera exclusiva para dicha entidad, sin que esta pueda garantizarle el empleo a largo plazo (Rentería, 2009). Se le exige comprometerse pero a su vez depender exclusivamente de sí mismo cuando se trata de sostenerse laboralmente.

Una cuarta paradoja tiene que ver con el control para organizar el trabajo vs. la acción sobre la contingencia (Rentería, 2009). Se le pide al trabajador que planee su trabajo, que se anticipe frente a las dificultades que pueden presentarse $y$ que no deje ningún cabo suelto, bajo un contexto cada vez más inestable y fluído, tal cual explica Bauman (2001) a través de su metáfora, donde finalmente dicho trabajador tiene que solucionar las cuestiones y salir avante lidiando con las contingencias que van emergiendo en el camino.

Como quinta paradoja se menciona una cada vez más tangible en este momento histórico referida por Malvezzi (2012), al respecto de la posibilidad de contar con múltiples canales de comunicación vs. el alejamiento de las comunidades/personas. El desarrollo tecnológico de las últimas décadas permite nuevas maneras de comunicarse, la telefonía celular, las aplicaciones computacionales y en general las redes sociales virtuales, se supone conducirían a que las personas están más comunicadas y en mayor contacto, sin embargo esta disponibilidad de canales se antepone al alejamiento del individuo de las personas y comunidades, a través de la reducción del contacto físico y la pérdida de importancia de lo presencial para relacionarse, estando en mayor interconexión, pero cada vez más alejados de los otros.

La realidad actual presenta a través de sus paradojas retos interesantes para efectos de lograr mayor comprensión sobre su naturaleza, que implica salir de las visiones lineales y pensar dicha realidad desde una perspectiva compleja (Urry, 2005), o al menos procurar identificar tendencias. Los cambios en el mundo del trabajo están siendo descritos en la literatura especializada por lo general de manera desconectada, y es preciso identificar tendencias como las siguientes para ayudar a percibir las conexiones entre los eventos que hoy en día se hacen cada vez más comunes: la tendencia a la competición, la tendencia a la tecnologización y la tendencia a la globalización (Malvezzi, 2012; Rentería, 2012). Estos aspectos influyen directamente sobre lo social, y por tanto también influyen en la organización de los procesos productivos, y sobre la manera en que las personas se relacionan con el trabajo.

La tendencia a la competición -en un contexto económico y político de acuerdo con Malvezzi (2012) - se refiere a la disputa por los recursos y por el poder, e implica la anulación de los otros. Bajo las formas en que se ha representado el capitalismo en las últimas décadas (Jamenson, 2011) las manifestaciones de la competición conllevan al desconocimiento de las capacidades del contrincante, aun si estas son mejores que las propias, tratando de eliminarlo del mercado.

La competición se vuelve tendencia al poner el énfasis sobre la calidad, entendida esta como la explotación de las competencias de los objetos, hechos y eventos, a través de la optimización de los procesos de adaptación, para responder a la productividad (Malvezzi, 2012); el poner la calidad como criterio principal de las relaciones mercantiles, lleva a descuidar los medios, en la relación medios-fines, donde entonces solo importa los resultados.

Al poner el foco en los fines y descuidar los medios, implica que cualquier camino para cumplir con los objetivos se considera válido, dejando de lado aspectos morales y éticos fuertemente arraigados décadas atrás. Bajo esta forma de razonar, las instituciones que indican los parámetros del comportamiento y lo correcto no son más necesarias -como la iglesia, la familia, la escuela, el estado-, ya que la regla (lo que se debe hacer o no) la impone entonces el logro de resultados -la calidad-. Nos encontramos en un momento histórico caracterizado por la pérdida de patrones exclusivos de racionalidad que dirijan los sistemas sociales (sistemas huérfanos, Malvezzi, 2012), lo que a su vez se relaciona con el debilitamiento de las instituciones modernas (Bendassolli, 2007) que por décadas sostuvieron el funcionamiento de lo social. 
Centrarse en la competición lleva al cumplimiento de los objetivos sin considerar al otro ni las consecuencias posteriores a largo plazo, basándose solo en la ejecución bajo los medios disponibles y más favorables para alcanzar las metas; bajo esta racionalidad, diversas cuestiones de la relación persona-trabajo terminan siendo tocadas.

La tecnologización, por su parte, se refiere a los avances tecnológicos que se caracterizan por ser cada vez más próximos entre ellos (avanza más rápido) y contar con incrementos cualitativos mayores. Esta tendencia se relaciona con dos aspectos que modifican la forma de las personas relacionarse con el trabajo. En primer lugar -asociado al desarrollo industrial producido desde finales del siglo XIX- se da el desplazamiento de la mano de obra hacia la máquina, reduciendo la importancia de lo humano para la producción de lo económico, y a su vez, se pierde el trabajo como valor económico para las personas (Bendassolli, 2007).

En segundo lugar, la tecnologización implica que se modifique la percepción de la relación tiempo-espacio. El contar con múltiples posibilidades para entender lo temporal y lo espacial, ha diversificado las formas de trabajar, pero al tiempo ha traído consecuencias de otro orden que influyen incluso en esferas como la familiar (donde se mezclan espacios y tiempos de trabajo y familia debido a posibilidades como el teletrabajo), y la salud de las personas que trabajan (al no poner límites por ejemplo a los horarios y a la carga que es posible asumir a través de la virtualidad). Con la existencia de lo virtual, se abre la puerta a modificar la percepción de cuestiones que incluso son -y seguirán siendo- tangibles, y que a su vez imponen límites naturales o marcos de referencia para la relación de las personas con el mundo, como lo es el cuerpo, que también se cansa y desgasta, y requiere tiempo y espacio para su recuperación, tiempos y espacios que en la virtualidad no están presentes porque en ese contexto son innecesarios (Soto, 1998).

La tendencia a la virtualización y su respectiva influencia en la relación espacio-tiempo, implica cambios en el trabajo (tanto para la persona como para las organizaciones), modificando en primer lugar el apoyo que se le da al individuo (ya que pareciera no requerir más presencia del otro para favorecer su desempeño); en segundo lugar modifica el apoyo que se le da al grupo; en tercer lugar se automatizan los procesos organizacionales; y finalmente cambian las formas de comunicación generales (Thach y Woodman, 1994, citados por Soto, 1998). De acuerdo con Soto, la virtualización modifica las maneras de proximidad entre las personas, influyendo también en las formas de agenciar, cambiando la relación de las personas con el trabajo.

La globalización por su parte puede entenderse como la tendencia a la interconexión entre los cambios y consecuencias acontecidos en lo social, donde todas las variables se afectan entre sí, y resulta imposible comprender la realidad de manera univariada o a través de únicas variables independientes. De la Dehesa (2002), Pizano (2002) y Castells (2003), indican que la globalización se relaciona con la modificación radical del orden social, y en particular con la occidentalización del mundo bajo la lógica del postcapitalismo (Castells, 2003).

Para Malvezzi (2000), la globalización implica una serie de dimensiones, además de la económica que es su cara más visible, donde cada vez es mayor la interdependencia entre las naciones alrededor del mundo, sin considerar su nivel de desarrollo; la caída económica de un país, puede mover la economía mundial. Para el autor, la globalización implica dimensiones ontológicas, sociológicas, antropológicas y psicológicas.

En su dimensión ontológica, la globalización implica la compresión de la relación espacio-tiempo, como fue mencionado anteriormente relacionada con la tecnologización donde se modifica o altera dicha relación. La dimensión sociológica implica imprevisibilidad en el funcionamiento de las sociedades, lo que puede llegar a afectar de manera radical e incluso a escala social mundial. La dimensión antropológica se relaciona con que en este momento de la historia los seres humanos se encuentran invadidos por múltiples referentes culturales y simbólicos que comunican distintos marcos de valores incluso contradictorios entre 
ellos (Malvezzi, 2000). Finalmente, el autor indica que la globalización implica además una dimensión psicológica, referida a la posibilidad de construir múltiples identidades en relación con el mundo, por la oportunidad de la virtualidad, y la ocupación de múltiples roles en diversos contextos, y espacios y tiempos iguales o diversos, sin que esto implique alteraciones de orden psíquico.

Las tendencias a la competición, tecnologización y globalización, como transversales a las formas de configurar lo social en este momento de la historia -y por tanto de re-institucionalización del trabajo-, tienen impactos en la relación personatrabajo, que pueden describirse como complejos y multicausados, por lo que no es fácil discernir la supraordinación entre ellos, pero que son importantes considerar para comprender el contexto social que rodea, condiciona e influye en la manera como las personas se relacionan con el trabajo.

Como impactos de las tendencias mencionadas se consideran los siguientes aspectos: 1) precarización del trabajo y cambio en el funcionamiento de las organizaciones; 2) cambios en las formas y manifestaciones del trabajo; 3) Cambio en la forma de representarse el ser humano al respecto del trabajo; 4) cambios en los criterios para la inclusión (y exclusión) y mantenimiento en el mercado de trabajo y la responsabilización del sujeto al respecto de su empleabilidad; 5) cambios en la configuración de la subjetividad y el lugar que el trabajo ocupa en ella incluyendo el establecimiento del contrato psicológico, la construcción de la carrera, la identidad, y la forma de representarse la agencia; y 6) cambios en la forma de gestionar el recurso humano al interior de las organizaciones. Se asume que esta lista y la siguiente explicación sobre los impactos o cambios en las relaciones de trabajo no implican el trato exhaustivo de los mismos, por desbordar el presente documento.

\section{La precarización del trabajo y el cambio en} el funcionamiento de las organizaciones: la precarización se refiere al empobrecimiento de las condiciones de trabajo que afectan el bienestar a corto y largo plazo del sujeto y que, de acuerdo con Castel (2000), se refleja en la incapacidad de la persona para planear su futuro, incluso en otras dimensiones de la vida distintas del trabajo como estudiar, establecerse con una pareja y pensar en tener hijos.

La precarización se apoya en la flexibilización de las regularidades propias del mundo del trabajo construidas hasta después de la mitad del siglo XX; el apoyo radica en la flexibilización de los aspectos legales que se generaron para preservar el bienestar del trabajador en décadas anteriores, y por la naturalización de una racionalidad que implica para el sujeto alto esfuerzo acompañado de incertidumbre respecto de su permanencia en el puesto de trabajo (e incluso en el mercado de trabajo en general), asociado esto a las tres tendencias mencionadas anteriormente.

Podría decirse que la precarización es una manifestación de la cada vez más común desprotección del ciudadano por parte del Estado-Nación, que se ha evidenciado a lo largo de las últimas décadas, relacionado también con el debilitamiento de las estructuras fuertes de la modernidad, según expresa Bendassolli (2007), y que trae implícita la idea de que el sujeto es responsable por su inserción y mantenimiento en el mercado de trabajo, idea que favorece el discurso de la responsabilización del sujeto (Beck y Beck, 2002).

La precarización además se fortalece por la búsqueda de competitividad por parte de las organizaciones, que al seguir estándares internacionales, privilegian los resultados por encima de los procesos de trabajo, y el bienestar de los trabajadores, amparados en legislaciones laborales que permiten estas formas de intermediación. Adicionalmente debe mencionarse el impacto de las multinacionales (industriales) que se mueven alrededor del mundo buscando mano de obra barata, y que promueven la división internacional de trabajo, a través de emplear con bajos salarios y condiciones mínimas a ciudadanos de países en vías de desarrollo, que, aunque contando con trabajo, siguen estando en la escala más baja en una comparación internacional de trabajadores.

La flexibilización en las organizaciones, estaría caracterizada retomando a Grimshaw, Marchington, Rubery y Willmott (2005), a través de la descentralización del poder, así como la tendencia 
a funcionar como red; cada vez es más probable el hecho de no saber dónde termina una organización y empieza otra, según indican los autores, debido a la tercerización de servicios, y donde las obligaciones con los empleados son un asunto más negociado que estable, garantizando con esto ambigüedad e incerteza en las relaciones laborales.

Lo anterior, de acuerdo con Castel (2000), cuestiona la función integradora del trabajo en la sociedad, manifestándose en el desmonte de las protecciones y garantías vinculadas al empleo. El autor identifica tres aspectos básicos de la situación actual que pueden relacionarse con la precarización del trabajo, en primer lugar lo que llama la desestabilización de los estables relacionada con la flexibilidad; en segundo lugar, la instalación de la fragmentación; y en tercer lugar, la exclusión de personas que resultan ser sobrantes en el mercado de trabajo actual.

Sobre la flexibilidad, el autor indica que es la medida que toman las organizaciones para reducir costos y seguirse manteniendo competitivos en el mercado de trabajo. Según expone Castel (2000), la flexibilidad puede ser interna o externa; la flexibilidad interna se refiere a exigir la adaptabilidad de los trabajadores y promover la polifuncionalidad como un aspecto que favorece el seguir enganchado en la organización. La flexibilidad externa es la tercerización de la mano de obra, subcontratando a organizaciones y/o personas para que se encarguen de procesos enteros, dedicándose la organización solo a sus funciones centrales.

Sobre la flexibilización interna, puede decirse que complejiza la forma en que las personas se relacionan con el trabajo, ya que no solamente se trata de un trabajo más abstracto -especialmente en cargos de mayor responsabilidad y que requieren mayor preparación no solo académica sino también en competencias-, sino que además tienen que responder en distintos frentes simultáneamente y enfrentarse a un mayor número de solicitudes laborales -probablemente más complejas-, considerando que al interior de cada labor a su vez deben asumir cambios importantes en las condiciones en las cuales se trabaja.
Al respecto de la flexibilización externa Grimshaw, Willmott y Rubery (2005), indican que cada vez es más común el establecimiento de relaciones interorganizacionales que procuran promover la competitividad de quienes las integran. Según los autores, el establecimiento de estas redes tiene como principales propósitos modificar la reputación de la organización en el mercado, reducir costos y mejorar el desempeño. Igualmente el establecer relaciones interorganizacionales trae implicaciones para las personas que participan de ellas, especialmente cuando deben desempeñar labores en organizaciones distintas a la que contrató inicialmente; de acuerdo con Grimshaw, Willmott y Rubery, el contrato psicológico, el compromiso y la discreción pueden verse afectados por los cambios en las relaciones de trabajo producto de estas redes.

En las organizaciones flexibilizadas se pueden generar relaciones intraorganizacionales, que implican los vínculos diversos con distintos trabajadores, trayendo implicaciones en las formas de establecer relaciones entre estos, así como de estos hacia la organización. Igualmente, desde la perspectiva del trabajador, pueden darse relaciones multiorganizacionales, entendiendo estas como sinónimo de multiempleo, donde Según Rubery, Earnshaw y Marchington (2005), se tocan aspectos centrales de la relación de las personas con el trabajo que se configuran de forma distinta en comparación con la relación de un único empleo; entre dichos aspectos se encuentra el nivel de control sobre el trabajo, la forma en que se conciben y se atienden los clientes, la equidad en el pago, la discriminación por raza y sexo, y la responsabilidad sobre la salud y cuidado de los trabajadores, entre otros, según mencionan los autores.

En resumen, la instalación de la fragmentación mencionada por Castel (2000), se manifiesta según Grimshaw, Marchington, Rubery y Willmott (2005) en las relaciones flexibles donde se realizan trabajos para varias organizaciones a la vez, o distintos trabajos para una misma organización, aunque asimismo los autores destacan que este tipo de trabajos favorecen la creatividad y el desarrollo de otras aptitudes. Estas formas de trabajo que implican fragmentación pueden com- 
plicar las relaciones entre grupos y niveles jerárquicos, favorece la responsabilización del sujeto por su salud y su bienestar en el trabajo, y puede conducir a reducir el compromiso y sentido de pertenencia con la organización, donde además la persona no cuenta con espacios satisfactorios para el establecimiento de relaciones interpersonales profundas por la volatilidad de sus contextos de trabajo; a su vez, dichas formas de trabajo se ven apoyadas en las leyes laborales de cada país (Díaz, Godoy y Stecher, 2005), donde se reducen las dificultades para que las organizaciones puedan realizar despidos e implementar contratos a tiempos cortos.

Kallinikos (2003), indica que la fragmentación tiene realmente su raíz en un aspecto mucho más transcendental que por ejemplo la misma globalización, y es en la desconfianza en las instituciones sociales y la tendencia a la individualización. Esta última entendida por el autor como la transferencia de la responsabilidad por las condiciones y contenido del trabajo de las instituciones al individuo (Kallinikos, 2003).

2. Los cambios en las manifestaciones y modalidades de trabajo: El presente momento histórico con las formas de reinstitucionalización descritas anteriormente, favorecen la aparición y fortalecimiento de otras manifestaciones y modalidades de trabajo. Al respecto de las manifestaciones, el mercado de trabajo mudó de centrarse en el sector industrial, a generarse múltiples trabajos en el sector servicios -tanto dentro de lo lucrativo, como de lo no lucrativo, y dentro de lo público y lo privado-, apoyado a su vez en la inmanencia ${ }^{1}$ y en la importancia adquirida por lo simbólico (discurso vs. Realidad), genera un mercado más abstracto, y donde se pueden crear valores de cambio que décadas atrás no se concebían como posibles (por ejemplo la venta de agua embotellada como una práctica cotidiana, un recurso al que se su-

1 Entendida de acuerdo con Malvezzi (2012) retomando a Déchaux, como la pérdida de los referentes centrales o únicos que indicaban cómo debe funcionar el mundo, pasando a la aparición -y aceptación- de la existencia de múltiples maneras de entenderlo y relacionarse con este. Esta tendencia a la inmanencia en la sociedad actualmente, también se relaciona con la introducción de racionalidades derivadas de la cientifización de lo social, que, a partir de incluir tratamientos positivistas, desmontaron los deónticos o de autoridad instaurados siglos atrás donde los aspectos de orden moral y éticos estaban en primer lugar. pone todas los seres humanos tienen derecho de acceder al menos a través de un acueducto).

Para Antunes (2001), se está dando una reducción del proletariado industrial, fabril, tradicional, manual, estable y especializado propio del esquema taylorista/fordista, con incremento del nuevo proletariado fabril y de servicios que se caracteriza por funciones de "intermediación, regulación, ordenación y normalización...”, donde se realizan "trabajos de tipo terapéuticos, pedagógicos, policiales, de aseguramiento y mediación de los contextos comunicacionales, si se quiere evitar daños para el orden de la sociedad adquisitiva" (p. 33), así como la aparición de trabajadores del tercer sector (Antunes, 2001), que da cuenta de las personas que realizan actividades comunitarias y asistenciales, muchos de ellos bajo trabajos precarizados, en organizaciones sin ánimos de lucro y ONG.

En este contexto, de un mercado de trabajo atravesado por lo simbólico, apoyado además en el desarrollo tecnológico y la globalización, se hacen posibles formas de trabajo inexistentes pocos años atrás sostenidos en la virtualización como los web manager, o el teletrabajo, donde no se hace necesaria una localización específica para trabajar y donde por lo general el trabajador cubre los costos ocultos que se ahorra la organización al permanecer en su hogar en el horario de trabajo como es el consumo de servicios públicos.

Por su parte, en términos de las modalidades de trabajo, aunque puede decirse que siempre han existido otras formas distintas de trabajar al empleo, antes de la era industrial, es el presente momento histórico el que favorece la presencia y fortalecimiento de modalidades como la prestación de servicios, el outsourcing, y los trabajos que cuentan con principios cooperativos, además de la aún presencia del empleo; todas estas modalidades en sus dinámicas de funcionamiento traen implicaciones diversas a los trabajadores (Rentería, 2001). Y exigen de la educación como espacio de socialización para el trabajo, otras formaciones a las que parece le cuesta dificultad responder; de alguna manera puede decirse que se sigue preparando para el empleo.

A lo anterior se suma otro fenómeno que cada vez tiene mayor crecimiento y es el trabajo in- 
formal, presente anteriormente en países en vías de desarrollo, pero que por aumento generalizado del desempleo en todo el mundo, se ha vuelto un espacio común en países considerados desarrollados. Incluso, el mercado informal puede ser una muestra de la diversificación en el mundo del trabajo, al encontrarse en este desde personas con baja escolaridad, hasta personas con niveles académicos superiores, así como encontrar trabajadores con bajos ingresos y condiciones precarias propias del subempleo, hasta personas ejecutando trabajos donde se gana más dinero que si se estuviesen en el mercado formal y con mejores condiciones que aquellas ofrecidas en este.

\section{El cambio en la forma de representarse el ser} humano al respecto del trabajo: la tendencia a la tecnologización, y el reemplazo de la mano de obra por la máquina, desplaza al hombre como elemento central para la realización del trabajo, modificando uno de los pilares del trabajo como institución moderna de acuerdo con Bendassolli (2007), al dejar de ser -exclusivamente visto como- generador de valor económico. A lo anterior se suma el aumento de la población en capacidad de trabajar (Malvezzi, 2012), que además cada vez está más cualificada, y la facilidad para deslocalizar organizaciones que para reducir costos, se trasladan a contratar mano de obra barata en países en vías de desarrollo.

Todo lo anterior favoreció la naturalización de asumir al ser humano, de acuerdo con Malvezzi (2012), como un hombre modular, perdiendo su dimensión de ser (completo), y valorizando de él solo algunos de sus aspectos, según sea indispensable para la tarea a realizar, donde las personas parece que se terminan percibiendo como seres desechables (Peralta, 2012). Además, asociado a este cambio se encuentra el hecho de que en las últimas décadas el crecimiento económico ya no depende de la mano de obra disponible, ni es condición suficiente para la creación de puestos de trabajo, así como tampoco produce automáticamente desarrollo social, y no representa necesariamente una barrera para la destrucción del desempleo.

Blanch (1996), plantea la cascada de realidades donde no se establecen más relaciones de cau- salidad entre situaciones que en los esquemas industriales propiamente dichos era inevitable relacionar. Dentro de estas realidades expone que la fuerza humana de trabajo ya no es la principal mercancía ni tampoco el medio fundamental de autorrealización/alienación personal, así como no determina decisivamente el valor de los bienes y servicios económicos, y las personas desempleadas ya no explican la dinámica del mercado laboral como sucedía en épocas anteriores. A lo dicho se suman observaciones como las de Offe (1992) al exponer que existe gran variación en las situaciones laborales acerca de "los ingresos, las calificaciones, la seguridad en el puesto de trabajo, la transparencia y el reconocimiento social, la carga de trabajo, las oportunidades de ascenso, las posibilidades de comunicación, la autonomía, etc." (p. 27), así como otros argumentos asociados presentados por autores como Luque, Gómez y Cruces (2000), al mencionar que existe un desajuste entre las personas y la oferta de puestos de trabajo, al nivel que el desempleo comienza a ser estructural "incluso en los países más avanzados" (p. 15), y que las tendencias del mundo del trabajo se conducen a un mercado dual donde existen personas con exceso de trabajo, y otros que precariamente lo hacen; probablemente las actividades que se realizan en estos últimos trabajos no requieren de alta cualificación, y los primeros implican tareas cada vez más elaboradas y de mayor responsabilidad.

Este mismo panorama lo recrea Teixeira (1997), al dar cuenta de cómo se han modificado los esquemas de trabajo desde los tiempos previos al nacimiento de la era industrial, hasta los tiempos actuales donde el presente “... puede ser comparado con el surgimiento del capitalismo de las primeras industrias" (p. 48), refiriéndose a las dificultades de humanizar el trabajo, y al desequilibrio entre la vida personal y la laboral de las personas que trabajan en la actualidad.

Una visión modular del ser humano al respecto del trabajo se asocia asimismo al culto a la inmediatez, que no deja tiempo para reflexionar y construir desde el raciocinio, exigiendo a las personas rapidez para actuar, induciendo a no reflexionar para responder a los fines, restándole 
importancia a los medios. Esto a su vez termina impactando los alcances de las acciones emprendidas por los sujetos, ya que por responder a la urgencia se realizan planeaciones a corto plazo para cumplir con la productividad, pero que no dejan mayor impacto al no tener continuidad, ni una visión global y a largo plazo de los fenómenos.

\section{Los cambios en los criterios para la inclusión (y} exclusión) y mantenimiento en el mercado de trabajo: En relación con lo anterior, si en el actual mundo del trabajo se privilegia el resultado más que el proceso, si cada vez son más las personas en capacidad de trabajar (además cualificadas), y los trabajos disponibles son aquellos de mayor exigencia en competencias porque los operativos están siendo reemplazados por las máquinas desde la tecnología, los criterios para lograr incluirse y mantenerse en el mercado de trabajo terminan modificándose, tornándose más exigentes y abstractos, y favoreciendo nuevos sistemas de exclusión. Estos elementos llevan nuevamente a visibilizar que la manera en que se está configurando lo social en relación al trabajo, favorece a la responsabilización del sujeto respecto de su empleabilidad.

Los criterios de inclusión y permanencia en el actual mercado de trabajo van desde aspectos explícitos determinados por las organizaciones como la cualificación -que de acuerdo con Franzoi (2006), pasó de ser un criterio indispensable para el desempeño adecuado del cargo, a ser un filtro para ingresar y mantenerse en el mercado de trabajo-, hasta criterios implícitos construidos socialmente, que sin ser realidades tangibles, tienen consecuencias reales en la relación de las personas con el trabajo, como por ejemplo la reducción del rango de edad para considerarse empleable, que empieza cada vez más tarde ${ }^{2}$, y termina más temprano en la vida de una persona ${ }^{3}$.

La cuestión problemática al respecto de estos criterios es el hecho que el mercado le exige a la persona ser cada vez más consciente de sí y sus actos, tener capacidad de reflexionar sobre sí

2 Teniendo con esto el incremento en el desempleo juvenil, representado principalmente en los países desarrollados.

3 Generando que cierto tipo de trabajadores altamente productivos queden por fuera del sistema de trabajo por estar por encima de dicho rango de edad, siendo aún altamente productivos. mismo, y pensarse como objeto (como mercancía) respecto a qué tan atractivo se es para el mercado, y cómo favorecer el serlo o seguirlo siendo para continuar inserto (Rentería y Enríquez, 2006). De acuerdo con Rentería y Malvezzi (2008) ni todas las personas están preparadas, ni todos los contextos sociales en que estas son socializadas favorecen dichos niveles de conciencia.

Adicional a lo anterior, se presenta la transnacionalización del capital -del sistema productivo-, donde las exigencias para los trabajadores cada vez son más homogéneas, aunque no hayan tenido las mismas oportunidades, ni se encuentren necesariamente listos ni dispuestos para asumirlas, y requieran de ajustes psicosociales de su parte (Rentería, 2005) que incluyen diversos tipos de estrategias (Entríquez y Rentería, 2007) para lograr posicionarse en el mercado de trabajo.

Las personas se enfrentan a otro tipo de exigencias para garantizar su supervivencia (Wilpert, 2009) en comparación con las de décadas anteriores donde la estabilidad (Munduate, 1997) y la constancia (Sennett, 2002) eran las características principales de las relaciones en el mercado de trabajo. Para las personas que trabajan en el presente, trabajar les implica mayor esfuerzo mental (Peiró, 2004); mayor flexibilidad para continuar insertos en el mercado y polifuncionalidad en las actividades realizadas (Gálvez, 2001); mayor capacidad para trabajar en equipo considerando la tendencia a depositar la gestión sobre los equipos (Malvezzi, 2000); mayor y mejor manejo de tecnología que les permita mantenerse actualizados y en contacto con el entorno (Peralta, 2012); apertura para la realización del trabajo indiferente del tiempo y del lugar, y estar dispuesto a realizar más de lo esperado para lograr destacarse entre otros trabajadores (Peiró, 2004). Pese a todo lo anterior, no es garantía para insertarse y mantenerse en el mercado de trabajo, dado que esta posibilidad no solo es configurada por el sujeto sino también por las instituciones, las organizaciones de trabajo, y las construcciones sociales que se hagan en torno a los criterios de quiénes pueden entrar y quedarse en el mercado (Rentería, 2005).

5. Los cambios en la configuración de la subjetividad y el lugar que el trabajo ocupa en ella 
incluyendo el establecimiento del contrato psicológico, la construcción de la carrera, la identidad, y la forma de los sujetos representarse la agencia: las actuales formas de re-institucionalización de trabajo favorecen lo que Bendassolli (2007) llama el debilitamiento de las estructuras fuertes de la modernidad, entre ellas del trabajo, por ya no constituirse como fuente principal de valor económico, principio moral, transmisor de ideología, contrato social -que sostiene la cooperación y solidaridad en la sociedad- y fuente principal de construcción de subjetividad.

En este sentido, y de acuerdo con la crítica que hace Offe (1992), existe una notable pluralidad sobre el hecho de trabajar donde incluso los mismos trabajadores están ampliamente diferenciados dependiendo del grado de influencia de la racionalidad utilizada en la división del trabajo. El autor afirma que lo único que tienen en común las personas que trabajan es que se encuentran articulados en una lógica capitalista donde todos venden su fuerza de trabajo como una mercancía, pluralidad que se explica con la existencia de mercados primarios y secundarios, de una economía semilegal y criminalizada, que también puede generar bienes y servicios, del crecimiento de posiciones intermedias o de clases mixtas que hacen un corte entre los trabajadores asalariados, y de las diferencias que hay entre las formas de trabajar de producción y de prestación de servicios.

Con base en lo anterior, Offe (1992) plantea que con todos los cambios en las relaciones en el mundo del trabajo ya no existe un significado que se represente como unitario, en este sentido, y según lo indicado también por Bendassolli (2007), la subjetividad ya no se construye (de manera exclusiva) a través del trabajo, o si se quiere pensar desde otra perspectiva, se construye de formas diversas a través del trabajo ${ }^{4}$. En este sentido entonces, aspectos como el contrato psicológico, la construcción de la carrera, la identidad, y la agencia también fueron afectados.

$4 \quad$ Como ocurre en sujetos que encuentran en el trabajo la fuente para el cumplimiento de funciones psicosociales que en décadas pasadas, por la forma de institucionalización del trabajo, no eran posibles.
En el caso del contrato psicológico, ya no se parte de considerar el compromiso con el trabajo como un hecho natural, como fuera representado en la era industrial, de acuerdo con la descripción de la ética antigua hecha por Sennett (2002) sobre la forma de asumir este tema en dicho momento histórico. El contrato psicológico que antes se daba por sentado, ahora debe construirse y alimentarse constantemente desde todas las partes involucradas, con la claridad incluso que puede (o va a) terminarse la relación. Lo anterior a su vez implica el cambio en la forma de concebir y gestionar (tanto por parte de las personas como de las organizaciones) temas asociados a la carrera, la identidad laboral, e incluso al proyecto de vida, y la importancia para la organización de no descuidar la subjetividad como aspecto central de la gestión de personas en organizaciones (Malvezzi, 2012).

Al respecto de la carrera, se evidencia cada vez más la diversidad en las formas de asumirla y construirla (Ribeiro, 2009a, 2009b). Desde una perspectiva tradicional se pensaba que se desarrollaba exclusivamente dentro de una organización, con movimientos ascendentes, y que implicaba etapas sucesivas incrementales, así como la noción de progreso en ese sentido. Considerando los cambios y las implicaciones de los mismos mencionados a lo largo del escrito al respecto de la configuración de lo social, la carrera hoy en día puede ser entendida como aquel curso en relación con la vida laboral que se puede desarrollar tanto dentro como fuera de las organizaciones (Ribeiro, 2011), o en varias organizaciones de forma consecutiva o paralela desde cualquier modalidad de trabajo, y donde la noción de progreso no está asociada necesariamente al ascenso, sino al cambio, incluyendo el cambio en sentido horizontal, y resaltando la importancia de aspectos como el aprendizaje y la experiencia.

Sobre la identidad, puede decirse que el trabajo no es ya necesariamente el referente principal en términos de predicados para describir a la persona, o describirse -o escribirse- a sí misma, respecto de la respuesta a la pregunta ¿quién soy? Se visibilizan otros órdenes entre los referentes identitarios que además se mueven a lo largo de la vida (Malvezzi, 2012) según los intereses de la persona, su 
biografía, y su adaptación al ambiente en que se desenvuelva ${ }^{5}$. En particular, la identidad profesional (laboral) se configura de tal manera, para que cuente con elementos que puedan ser mutables para responder a las demandas de los múltiples roles y de las demandas del cotidiano, sin necesidad de desestructurar el sujeto (Malvezzi, 2000).

Al respecto de la agencia, en este momento histórico los sujetos se enfrentan a un mundo de trabajo multivariado, de rápida mudanza y que es poco controlable. Los planes mentales que lleva a cabo el ser humano dependen de la forma en que perciba la relación medios-fines (Malvezzi, 1988), que se ve influida por las características actuales del mundo del trabajo, poniendo límites o definiendo alcances para las acciones del sujeto.

Puede decirse que el impacto al respecto de la agencia, es el constreñimiento de los rangos de libertad de la acción, donde además se actúa con mayor incertidumbre y con menor certeza de conseguir el impacto deseado según el curso de acción emprendido; es decir, las variables están más intrincadas e interdependientes, por lo que ahora menos que nunca es posible calcular concretamente las consecuencias de un curso de acción introducido; incluso las consecuencias pueden llegar a ser contrarias a los objetivos perseguidos. De acuerdo con Caldwell (2006) dependiendo del contexto y momento histórico, el entendimiento de la agencia, está atravesado por un flujo que oscila entre lo fijo y lo móvil, entre el determinismo y el voluntarismo, flujo que ahora implica mayor oscilación.

6. Los cambios en la forma de gestionar el recurso humano al interior de las organizaciones: considerando todos los elementos expuestos, la forma de gestionar el recurso humano se impacta radicalmente en aras a responder a los cambios en la relación hombre-trabajo, y orientarse a favorecer no solo de la productividad, sino también del bienestar y la autorrealización de los trabajadores. La gestión de recursos humanos afronta dificultades y desafíos al verse obligada a lidiar con

$5 \quad$ Esto también asociado a la tendencia, a la inmanencia, que favorece o exige la elección de dichos referentes para que el sujeto se configure a sí mismo, por ya no existir referentes principales o únicos que guíen sobre cómo debe construirse. Cada vez es más frecuente que se le exija al sujeto que se encargue de la configuración de su identidad profesional. la convivencia de distintas formas de relacionarse con el trabajo de manera paralela, buscando además no solo construir, sino también mantener el contrato psicológico, sin contar con las seguridades (laborales y simbólicas) que se podían ofrecer anteriormente desde la organización.

Para lograr lo anterior es necesario ampliar las maneras de concebir la relación Gestión de Recursos Humanos y performance organizacional, pasando de la descripción a la comprensión del fenómeno de acuerdo con Fleetwod y Hesketh (2010), y poniendo el énfasis en la conceptualización para comprender el fenómeno más que en la técnica, ya que esta no es el fin, sino el medio para el entendimiento de dicho fin. De acuerdo con los autores -en una sociedad que tiende a la inmanencia-, la gestión de recursos humanos puede ser leída como resistente a este cambio, por procurar respuestas de autoridad, trascendentales que finalmente no logran abarcar la complejidad del fenómeno.

Puede decirse entonces que la gestión de personas en este momento histórico, implica repensar las formas en que se entiende la relación persona-trabajo, y comprender que está atravesada por raíces mucho más profundas de orden social, que no pueden ser desconocidas al momento de intervenir o tomar decisiones que afecten a las personas que trabajan, ya sea desde contextos organizacionales, o incluso desde lo jurídico, lo económico y lo legal, considerando que hoy en día el mundo de los negocios influye en gran medida en el funcionamiento de lo social (Rentería, 2008).

\section{Conclusiones}

Los cambios acontecidos en el mercado de trabajo se refieren concretamente a las formas en que se configuran las relaciones de trabajo, y no al sistema de fondo que sostiene el mercado (o mercados) laboral(es), que es el capitalismo.

Las paradojas fruto de las contradicciones capitaltrabajo se van agudizando con la complejización del mundo del trabajo, implicando exigencias contradictorias, mensajes con múltiples interpretaciones, y un panorama cada vez más incierto, 
que señala hacia las personas que trabajan, la responsabilidad por mantenerse vigentes cuenten o no con los repertorios para hacerlo.

Los cambios en las relaciones de trabajo traen implicaciones para las organizaciones y para las personas, por efecto de estas responder a las tendencias de la competición, tecnologización y globalización. La forma de gestionar personas dentro de las organizaciones se modificó, y la relación que las personas establecen con el trabajo terminó siendo a su vez influida. Tanto las organizaciones como las personas que trabajan requieren, más que nunca, promover procesos reflexivos que superen la exigencia de inmediatez que no deja tiempo para pensar en un momento histórico fluido como el presente, y que se orienten hacia la productividad de la mano de la promoción de la calidad de vida en un sentido ampliado, desbordando lo estrictamente económico. Sin este tipo de propósitos seguirá creciendo la sensación de caoticidad con el tinte catastrófico que ha caracterizado el mercado de trabajo en las últimas dos décadas.

\section{Referencias}

Antunes, R. (1999). 0 mundo precarizado do trabalho e seus significados. Cadernos de Psicologia social do trabalho, 2(1), 55-59.

Antunes, R. (2001). A clase-que-vive-do-trabalho. A forma de ser da clase trabalhadora hoje. Em Os sentidos do trabalho. Ensaio sobre a afirmação e a negação do trabalho, 101-117. São Paulo: Boitempo.

Bauman, Z. (2001). Modernidad líquida. Río de Janeiro: Jorge Zahar Editor.

Beck, U. y Beck-G. (2002). Individualization. UK: Sage.

Bendassolli, P. (2007). Trabalho e identidade em tempos sombrios. São Paulo: Ideias e letras.

Blanch, J. M. (1996). Psicología Social del Trabajo. En J. L. Álvaro, A. Garrido, y J. R. Torregrosa (eds.). Psicología Social Aplicada, 87-117. Madrid: McGraw Hill.
Caldwell, R. (2006). Agency and change. London: Routledge.

Castel, R. (2000). As transformações da questão social. Em R. Castel; L. E. Wanderley y M. Belfiore-Wanderley (eds). Desigualdade e a questão social, 235-272. São Paulo: Educ.

Castells, M. (2003). Prólogo. En F. Calderón (ed.). ¿Es sostenible la globalización en América Latina? debates con Manuel Castells. La globalización y América Latina: asignaturas pendientes. Santiago de Chile: Fondo de Cultura Económica.

De la Dehesa, G. (2002). Comprender la globalización. Madrid: Alianza Editorial.

Diaz, X., Godoy, L., y Stecher, A. (2005). Significados del trabajo, identidad y ciudadanía. La experiencia de hombres y mujeres en un mercado laboral flexible. Santiago de Chile: Purísima 353 Recolleta. Recuperado el 8 de octubre de 2010 de http://www.cem.cl/pdf/ cuaderno3.pdf

Enríquez, A., y Rentería, E. (2007). Estrategias de aprendizaje para la empleabilidad en el mercado del trabajo de profesionales recién egresados. Universitas psychologica, 6(1), 89103. Recuperado el 5 de mayo de 2008 de http://www.scielo.unal.edu.co/scielo.php? script=sci_arttext\&pid=S1657-92672007000 100010\&lng=pt\&nrm=

Fleetwod, S., y Hesketh, A. (2010). Explaining the performance of HRM, 3-59. UK: Cambridge University Press.

Franzoi, N. L. (2006). Profissão e profissionalização: aspectos conceituais e históricos. Em: Entre a formação e o trabalho: trajetórias e identidades profissionais, 23-52. Porto Alegre: UFRGS.

Gálvez, T. (2001). Taller sobre las nuevas modalidades del empleo. Recuperado el 25 de septiembre de 2008 de http://www.eclac.cl/ deype/mecovi/docs/TALLER12/12.pdf 
Gergen, K. (1991). El yo saturado. Dilemas de identidad en el mundo contemporáneo. Barcelona: Ediciones Paidós.

Grimshaw, D., Marchington, M., Rubery, J., y Willmott, H. (2005). Introduction: Fragmenting work across organizational boundaries. En M. Marchington, D. Grimshaw, J. Rubery, y $\mathrm{H}$. Willmott (eds.). Fragmenting work. Blurring organizational boundaries and disordering hierarchies, 1-38. New York: Oxford University Press.

Grimshaw, D., Willmott, H., y Rubery, J. (2005). Inter-organizational networks: Trust, power, and the employment relationship. In M. Marchington, D. Grimshaw, J. Rubery, y H. Willmott (eds.). Fragmenting work. Blurring organizational boundaries and disordering hierarchies, 39-62. New York: Oxford University Press.

Jamenson, F. (2011). Representing capital. A commentary on volumen one. London/New York: Verso.

Kallinikos, J. (2003). Work, human agency and organizational forms: an anatomy of fragmentation. Organization studies, 24(4), 595-618. Recuperado el 12 de junio de 2009 de http:// personal.lse.ac.uk/kallinik/pdf/KallinikosOrgStud.pdf

Luque, P. J., Gómez, T., y Cruces, S. J. (2000). El trabajo: fenómeno psicosocial. En C. Guillen y Guil, R. (eds.). Psicología del trabajo para relaciones laborales, 147-163. Madrid: McGraw Hill.

Malvezzi, S. (1988). The man-work relationship and organizational change. An approach to the humanization of work. PhD Thesis. Lancaster: Lancaster University.

Malvezzi, S. (1999). Empregabilidade e carreira. Cadernos de psicologia social do trabalho, 2(1), 55-72.

Malvezzi, S. (2000). Psicologia Organizacional. Da administração científica à globalização: uma história de desafios. Em C. Machado, V. Melo y M. Santos (eds.). Interfases da psicologia, 313-326. Portugal: Universidade de Évora.

Malvezzi, S. (2012). Seminario O homem e o Trabalho na Administração. Instituto de psicologia, postgraduação. Brasil: Universidade de São Paulo.

Munduate, L. (1997). Psicología social de la organización. Madrid: Ediciones Pirámide.

Offe, C. (1992). ¿Es el trabajo una categoría sociológica clave? En La sociedad del trabajo, 17-51. Madrid: Alianza Editorial.

Peiró, J. (2004). El sistema de trabajo y sus implicaciones para la prevención de riesgos psicosociales en el trabajo. Universitas Psychologica, 3(2), 179-186.

Peralta, M. C. (2012). Significados, cambios y contexto actual de trabajo. Estudio interpretativo. Divers: Perspect. Psicol., 8(1), 165-182. Recuperado el 20 de enero de 2013 de http:// revistas.usta.edu.co/index.php/Diversitas/ article/view/372/329

Pizano, D. (2002). Globalización: Desafíos y oportunidades. Bogotá: Alfaomega.

Rentería, E. (2001). El modelo educativo tradicional y los perfiles de competencias según las modalidades y tendencias del trabajo actuales. En Vinculación universidad-empresa a través del postgrado. Pautas y lineamientos, 51-60. Salamanca: AUIP. Recuperado el 2 de marzo de 2005 de http://objetos.univalle.edu. co/files/El_modelo_educativo_tradicional_y_ los_perfiles_de_competencia.pdf

Rentería, E. (2005). Empleabilidad: una lectura psicosocial. Revista de Estudios del Trabajo en Colombia, 1(1), 1-14. Recuperado el 5 de agosto de 2007 de http://objetos.univalle. edu.co/files/Empleabilidad_una_lectura_psicosocial.pdf

Rentería, E. (2008). Nuevas Realidades Organizacionales y del mundo del trabajo: Implicaciones para la construcción de la identidad o del sujeto. Informes Psicológicos, 10, 6580. Recuperado el 25 de septiembre de 2009 
de http://www.upb.edu.co/pls/portal/docs/ PAGE/GPV2_UPB_MEDELLIN/PGV2_M030_ PREGRADOS/PGV2_M030040020_PSICOLOGIA/PGV2_M030040020110_REVISTA/PGV2 M030040020110040_REVISTA10/PAGES\%20 FROM\%2OINFORMES\%20SICOLOGICOS\%20NUMERO\%2010\%20\%20AERT\%20NO\%204.PDF

Rentería, E. (2009). De Recursos Humanos a la Psicología Organizacional y del trabajo: Reflexiones a la luz de las realidades actuales del mundo del trabajo. En: C. Aguilar y E. Rentería (eds.). Psicología del trabajo y de las organizaciones. Reflexiones y experiencias de investigación, 25-51. Bogotá: Publicaciones Universidad Santo Tomás.

Rentería, E. (2012). Seminario de reflexiones teóricas en Psicología del trabajo y las organizaciones. Instituto de Psicología. Cali, Colombia: Universidad del Valle.

Rentería, E., y Enríquez, A. (2006). ¿Usted mismo S.A.? 0 el posicionamiento profesional en el mercado de trabajo. Reflexiones desde el marketing y el comportamiento del consumidor. Psicología desde el Caribe, 18, 139-160. Recuperado el 14 de diciembre de 2007 de http://objetos.univalle.edu.co/files/Usted_ mismo_sa.pdf

Rentería, E., y Malvezzi, S. (2008). Empleabilidad, cambios y exigencias psicosociales en el trabajo. Universitas Psychologica, 7(2), 319-334. Recuperado el 2 de febrero de 2010 de http://www.scielo.org.co/pdf/rups/v7n2/ v7n2a02.pdf

Revilla, J. C. (2003). Los anclajes de la identidad personal. Athenea digital, 4, 1-14. Recuperado el 2 de febrero de 2010 de http:// redalyc.uaemex.mx/src/inicio/ArtPdfRed. jsp?iCve $=53700404$

Ribeiro, M. A. (2009a). Carreira: transformações de uma concepção na teoria e na prática. Em: Psicologia e gestão de pessoas: reflexões críticas e temas afins (ética, competência e carreira, 119-159. São Paulo: Vetor.

Ribeiro, M. A. (2009b). A trajetória da carreira como construção teórico-prática e a propos- ta dialética da carreira psicossocial. Cadernos de Psicologia Social do Trabalho, 12(2), 203-216. Recuperado el 5 de abril de 2012 de http://www.revistasusp.sibi.usp.br/pdf/cpst/ v12n2/a06v12n2.pdf

Ribeiro, M. A. (2011). As formas da estruturação da carreira na contemporaneidade: interfaces e articulações teórico-técnicas entre a Psicologia Organizacional e do Trabalho e a Orientação Profissional. Em: Zanelli, J. C.; Silva, N. y Tolfo, S. R. (orgs.). Processos psicossociais nas organizações e no trabalho, 305-325. São Paulo: Casa do Psicólogo.

Rubery, J., Earnshaw, J., y Marchington, M. (2005). Blurring the Boundaries to the employment relationship: From single to multiemployer relationships. In M. Marchington, D. Grimshaw, J. Rubery, y H. Willmott (eds.). Fragmenting work. Blurring organizational boundaries and disordering hierarchies, 6388. New York: Oxford University Press.

Sennett, R. (2002). La corrosión del carácter. Consecuencias personales del trabajo en el nuevo capitalismo. Barcelona: Editorial Anagrama.

Sotto, R. (1998). The virtualization of the organizational subject. In: R. Chia (ed.). Organized worlds: Exploration in technology and organization with Robert Cooper. London: Routledge.

Teixeira, J. E. (1997). A busca do elo perdido na qualidade de vida no trabalho. Em: M. Do Carmo y M. Mazzilli (eds.). Empregabilidade e educação. Novos caminhos no mundo do trabalho, 43-57. São Paulo: Educ. Editora.

Urry, J. (2005). The complexity turn. Theory culture \& society, 22(5), 1-14. London: Sage.

Wilpert, B. (2009). Impact of globalization on human work. Safety Science, 47, 727-732. Recuperado el 5 de abril de 2012 de http:// www.choixdecarriere.com/pdf/6573/2010/ Wilpert2009.pdf 